\title{
Quantum ultra-walks: Walks on a line with hierarchical spatial heterogeneity
}

\author{
Stefan Boettcher 1 \\ Department of Physics, Emory University, Atlanta, Georgia 30322, USA
}

(Received 2 December 2019; accepted 18 June 2020; published 30 June 2020)

\begin{abstract}
We discuss the model of a one-dimensional, discrete-time walk on a line with spatial heterogeneity in the form of a variable set of ultrametric barriers. Inspired by the homogeneous quantum walk on a line, we develop a formalism by which the classical ultrametric random walk as well as the quantum walk can be treated in parallel by using a "coined" walk with internal degrees of freedom. For the random walk, this amounts to a second-order Markov process with a stochastic coin, better known as an (anti-)persistent walk. When this coin varies spatially in the hierarchical manner of "ultradiffusion," it reproduces the well-known results of that model. The exact analysis employed for obtaining the walk dimension $d_{w}$, based on the real-space renormalization group (RG), proceeds virtually identically for the corresponding quantum walk with a unitary coin. However, while the classical walk remains robustly diffusive $\left(d_{w}=\frac{1}{2}\right)$ for a wide range of barrier heights, unitarity provides for a quantum walk dimension $d_{w}$ that varies continuously, for even the smallest amount of heterogeneity, from ballistic spreading $\left(d_{w}=1\right)$ in the homogeneous limit to confinement $\left(d_{w}=\infty\right)$ for diverging barriers. Yet for any $d_{w}<\infty$ the quantum ultra-walk never appears to localize.
\end{abstract}

DOI: 10.1103/PhysRevResearch.2.023411

\section{INTRODUCTION}

Discrete-time quantum walks (QWs) [1] have captured the imagination of researchers in recent times. Their appreciation started at least with the quadratic gain over any classical search achieved by Grover's quantum search algorithm [2], with QWs as the main "diffusing" operation to spread information throughout an idealized memory. This was followed by the realization that a coined $\mathrm{QW}$ [3-5] in discrete time, distinctly from continuous-time alternatives [6], can bring such a gain also to more realistic two-dimensional geometries [up to smaller, logarithmic corrections to the leading $O(\sqrt{N})$ algorithmic complexity in problem size $N]$. Ever since, QWs have become objects of intense study $[1,7,8]$, beyond their algorithmic interest, on various geometries, not least of all on the one-dimensional (1D) line [9-13]. In simple geometries like lattices [14], fractals [15-17], and hypercubes [18], many of the features, such as ballistic spreading [9,13,19] and localization effects $[13,20-23]$ that distinguish QWs from classical random walks (RWs), are readily analyzed mathematically. These studies have spawned experimentally realizations to demonstrate transport and localization in QWs [24-30] and their search properties [31-34] that may become the foundation of future, controlled quantum computations.

The above-mentioned studies of solvable QWs on the line are all based on spatially homogeneous coins. Only a few examples exist concerning the dynamics of walks in heterogeneous but unitary environments, i.e., using coins

Published by the American Physical Society under the terms of the Creative Commons Attribution 4.0 International license. Further distribution of this work must maintain attribution to the author(s) and the published article's title, journal citation, and DOI. that vary extensively with location quasiperiodically [35] or are drawn from some random ensemble [22], each yielding analytical insights only into localization properties in some limits. Here we discuss a 1D walk in which coins possess a strong spatial variation but with a hierarchical repetition of coins, which we shall call the quantum ultra-walk. It is inspired by classical models of diffusion over an ultrametric arrangement of barriers [36-41] that was meant to describe ultra-slow relaxation. Among other things, ordinary diffusion in such a hierarchy proved to be quite robust, and the degree of heterogeneity had to reach a certain threshold before a walk became subdiffusive.

Using a real-space renormalization group ( $\mathrm{RG})$ [42], we can analytically determine the walk dimension $d_{w}$ [43], characterizing the asymptotic scaling variable $x / t^{1 / d_{w}}$ (or pseudovelocity [19]) for the walk, in closed form for a parameter that determines the relative strength of barriers. For example, $d_{w}$ describes the anomalous spread of the wave function with time in terms of the mean-square displacement, $\left\langle x^{2}\right\rangle \sim t^{2 / d_{w}}$ . Classically, $d_{w}$ also determines how recurrent a walk is; i.e., if the spatial (or fractal) dimension $d_{f}$ is larger than $d_{w}$, a walker might miss an arbitrarily close site forever, or might not return to a previously visited site. This connection is known as Pólya's recurrence theorem [44]. So the domain covered by such a walk is quite porous, while, in turn, that domain is almost certainly compact for $d_{f}<d_{w}$. The RG has been previously employed to obtain $d_{w}$ for QWs with a homogeneous coin in various fractal geometries $[17,45]$ and to elucidate the complexity of Grover's search algorithm in terms of the spectral dimension $d_{s}$ of the search space [5]. After a brief review of the RG for the homogeneous walk, we demonstrate our procedure first by rederiving the classical result in an alternative manner by using a secondorder Markov process $[46,47]$. It mimics the coined QW in all but the final step of the analysis while using a stochastic 
instead of a unitary coin [48]. Despite these parallels, quantum effects clearly assert themselves in the final analysis and, thus, in the behavior obtained for $d_{w}$.

This paper is organized as follows: Sec. II briefly reviews the simple (homogeneous) walk on a line. In particular, we introduce the dynamic equation that describes the evolution of the discrete-time walk, classical or quantum, and its RG treatment. In Sec. III we develop the RG for the case of a hierarchical dependence of such coins. In Sec. IV we choose a hierarchy of stochastic coins to derive the familiar classical result, Eq. (26). In Sec. V we then derive the solution for a corresponding hierarchy of unitary coins. We conclude with a discussion of our results in Sec. VI.

\section{BACKGROUND ON WALKS}

\section{A. Analytic properties of the master equation}

The time evolution of walks is governed by the discretetime master equation [48]

$$
|\Psi(t+1)\rangle=\mathcal{U}|\Psi(t)\rangle,
$$

with propagator $\mathcal{U}$. This propagator is a stochastic operator for a classical, dissipative RW. But in the quantum case it is unitary and, thus, reversible. Then, in the discrete $N$-dimensional site basis $|x\rangle$ of some network, the probability density function (PDF) is given by $\rho(x, t)=\psi_{x, t}=\langle x \mid \Psi(t)\rangle$ for RWs, or by $\rho(x, t)=\left|\psi_{x, t}\right|^{2}$ for QWs.

Assuming that we possess the eigensolutions for the propagator, $\mathcal{U} \phi_{j}=u_{j} \phi_{j}$ with eigenvalues $u_{j}$ and an orthonormal set of eigenvectors $\phi_{j}(x)$, then the formal solution of Eq. (1) becomes $\psi_{x, t}=\sum_{j} a_{j} u_{j}^{t} \phi_{j}(x)$. For a stochastic $\mathcal{U}$, aside from the unique $(+1)$ eigenvalue of the stationary state, the remaining eigenvalues have $\left|u_{j}\right|<1$; thus, according to Eq. (1), the dynamics is uniquely determined by $\rho(\vec{x}, t) \sim e^{-t / \tau}$ for large times $t$ with $\tau=-1 / \ln \max _{j}\left\{\left|u_{j}\right|<1\right\}$. In turn, for unitary $\mathcal{U}$, all eigenvalues are unimodular, $\left|u_{j}\right|=1$ for all $j$, such that $u_{j}=e^{i \theta_{j}}$ with real $\theta_{j}$. A discrete Laplace transform (or "generating function") [49] of the site amplitudes

$$
\bar{\psi}_{x}(z)=\sum_{t=0}^{\infty} \psi_{x, t} z^{t}
$$

has all its poles — and hence those for $\bar{\rho}(x, z)$-located right on the unit circle in the complex $z$ plane [50],

$$
\bar{\rho}(x, z) \propto \prod_{j, l}\left[1-z e^{i\left(\theta_{j}-\theta_{l}\right)}\right]^{-1} .
$$

For the stochastic propagator, these poles are located at $z_{j}=$ $1 / u_{j}$, accordingly, typically along the real- $z$ axis with $z_{j}>1$. These facts will prove significant for the interpretation of the $\mathrm{RG}$ results in Sec. V.

\section{B. Asymptotic scaling for walks}

For RWs, the probability density $\rho(\vec{x}, t)$ to detect a walk at time $t$ at site $\vec{x}$, a distance $x=|\vec{x}|$ from its origin, obeys the scaling collapse with the scaling variable $x / t^{1 / d_{w}}$,

$$
\rho(\vec{x}, t) \sim t^{-\frac{d_{f}}{d_{w}}} f\left(x / t^{\frac{1}{d_{w}}}\right),
$$

where $d_{w}$ is the walk dimension and $d_{f}$ is the fractal dimension of the network [51]. On a translationally invariant lattice of any spatial dimension $d\left(=d_{f}\right)$, it is easy to show that the walk is always purely "diffusive," $d_{w}=2$, with a Gaussian scaling function $f$, which is the topic of many classic textbooks on RWs and diffusion [47,52]. The scaling in Eq. (4) still holds when translational invariance is broken or the network is fractal (i.e., $d_{f}$ is noninteger). Such "anomalous" diffusion with $d_{w} \neq 2$ may arise in many transport processes $[49,51,53]$. Thus, the determination of $d_{w}$ provides fundamental insights into the physics of the spreading dynamics of a walk.

For QWs on ordinary lattices [54], Eq. (4) generically holds with $d_{w}=1$, indicating a "ballistic" spreading of a QW from its origin. This value has been obtained for various versions of one- and higher-dimensional QWs, for instance, with so-called weak-limit theorems [8,10,54-56]. The RG for a discrete-time QW with a coin $[17,48,50,57]$ was developed to expand the analytic tools to understand QWs, say, for networks that lack translational symmetries. This RG provides [45] principally similar results as in Eq. (4) in terms of the asymptotic scaling variable $x / t^{1 / d_{w}}$ (or pseudovelocity [19]), whose existence allows us to collapse all data for the probability density $\rho(\vec{x}, t)$, aside from oscillatory contributions ("weak limit").

\section{Coined walks on the line}

For nearest-neighbor transitions on a line, the propagator referred to in Eq. (1) becomes

$$
\mathcal{U}=\sum_{x} A_{x}|x+1\rangle\left\langle x\left|+B_{x}\right| x-1\right\rangle\left\langle x\left|+M_{x}\right| x\right\rangle\langle x|,
$$

where $A_{x}, B_{x}$, and $M_{x}$ specify the (possibly positiondependent) hopping operators for transitions to the left, right, or same site, on leaving from a site $x$. RW merely requires local conservation of probability, $A_{x}+B_{x}+M_{x}=1$, and could be satisfied with a scalar Bernoulli coin $p$, such that $A=p$, $B=1-p$, and $M=0$, for a homogeneous walk, for example. In contrast, conservation of probability for $\rho(x, t)=\left|\psi_{x, t}\right|^{2}$ in a $\mathrm{QW}$ demands unitary propagation, $\mathbb{I}=\mathcal{U}^{\dagger} \mathcal{U}$, which imposes on the "coin space" the conditions $\mathbb{I}_{r}=A_{x}^{\dagger} A_{x}+B_{x}^{\dagger} B_{x}+$ $M_{x}^{\dagger} M_{x}, A_{x}^{\dagger} B_{x-2}=0$, and $0=A_{x}^{\dagger} M_{x+1}+M_{x}^{\dagger} B_{x+1}$. For nontrivial choices satisfying $A_{x}^{\dagger} B_{x+2}=0$, this algebra requires at least $r=2$-dimensional hopping matrices. It is common $[1,8]$ to construct the propagator as a combination of a unitary $r \times r$ coin-matrix $\mathcal{C}_{x}$, acting on each site, and a shift operator $\mathcal{S}$. The coin mixes the components of $\psi_{x, t}$ locally while the shift is represented by a set of matrices $S^{(j)}$ connecting neighboring sites, providing for a subsequent transfer in each direction. In this manner, coin and spatial degrees of freedom become entangled.

For the propagator in Eq. (5), this suggests the choice of $A_{x}=S^{A} \mathcal{C}_{x}, B_{x}=S^{B} \mathcal{C}_{x}$, and $M_{x}=S^{M} \mathcal{C}_{x}$ with $S^{A}+S^{B}+$ $S^{M}=\mathbb{I}_{r}$. The simplest form, $r=2$, the degree of each site on the line, does not allow for self-loops $\left(S^{M}=0, M_{x}=0\right)$ but shifts upper (lower) components of each $\psi_{x, t}$ to the right (left) using the projectors

$$
S^{A}=\left[\begin{array}{ll}
1 & 0 \\
0 & 0
\end{array}\right], \quad S^{B}=\left[\begin{array}{ll}
0 & 0 \\
0 & 1
\end{array}\right] .
$$


Then it is easy to show that the unitarity conditions above are satisfied for arbitrary unitary coins $\mathcal{C}_{x}$.

\section{Renormalization of walks on a line}

The homogeneous RW or QW on a line, as defined above, is readily solved to find $\rho(x, t)$ via a Fourier transform (see Ref. [1], for example). However, many geometries lack translational invariance, in which case other methods need to be devised. One such method is the real-space RG [42], which is particularly effective for hierarchical, recursively defined systems. For the following, it is thus instructive to review the $\mathrm{RG}$ for a homogeneous walk on the line, which is trivially recursive.

To obtain the RG recursions, the master equation (1) is projected into $|x\rangle$ space with $\mathcal{U}$ as given in Eq. (5). Spatial homogeneity means that the hopping operators are $x$ independent. Time is eliminated by applying the generating function defined in Eq. (2), such that the master equation becomes

$$
\bar{\psi}_{x}=z M \bar{\psi}_{x}+z A \bar{\psi}_{x-1}+z B \bar{\psi}_{x+1} .
$$

For simplicity, we consider initial conditions (ICs) localized at a single site $x_{0}, \psi_{x, t=0}=\delta_{x, x_{0}} \psi_{\mathrm{IC}}$. Eliminating $\bar{\psi}_{x}$ for all sites for which $x$ is an odd number and setting $x \rightarrow x / 2$, the master equation reveals itself to be self-similar in form by appropriately redefining the renormalized hopping operators $A, B, M$. To see this, we write for sites adjacent to any even site $x$ [48]:

$$
\begin{aligned}
\bar{\psi}_{x-1} & =M \bar{\psi}_{x-1}+A \bar{\psi}_{x-2}+B \bar{\psi}_{x}, \\
\bar{\psi}_{x} & =M \bar{\psi}_{x}+A \bar{\psi}_{x-1}+B \bar{\psi}_{x+1}+\delta_{x, x_{0}} \psi_{\mathrm{IC}}, \\
\bar{\psi}_{x+1} & =M \bar{\psi}_{x+1}+A \bar{\psi}_{x}+B \bar{\psi}_{x+2} .
\end{aligned}
$$

Solving this linear system for the inner site $x$ yields $\bar{\psi}_{x}=$ $M^{\prime} \bar{\psi}_{x}+A^{\prime} \bar{\psi}_{x-2}+B^{\prime} \bar{\psi}_{x+2}+\delta_{x, x_{0}} \psi_{\mathrm{IC}}$, leaving no effect on the $\mathrm{ICs}$, but requiring the (nonlinear) RG recursions:

$$
\begin{gathered}
A^{\prime}=A(\mathbb{I}-M)^{-1} A, \\
B^{\prime}=B(\mathbb{I}-M)^{-1} B, \\
M^{\prime}=M+A(\mathbb{I}-M)^{-1} B+B(\mathbb{I}-M)^{-1} A .
\end{gathered}
$$

Physically, it expresses the effective behavior of a system in which every other site had been coarse-grained out in terms of the renormalized (primed) hopping operators, which now represent transitions over twice the distance of their (unprimed) priors. A recursive application of Eq. (9) reveals the asymptotic scaling of the walk, as given by Eq. (4), near the stationary ("fixed") points (FPs) [49]. Linearizing the nonlinear system of RG recursions, such as Eq. (9), around their FPs provides a Jacobian matrix whose eigenvalues relate the asymptotic behavior in space and time of the master equation in Sec. II A. While the eigenvalues of the propagator determine the location of poles of $\bar{\rho}(x, z)$ in Eq. (3), the Jacobian eigenvalues here determine how these poles move in the complex $z$ plane under rescaling space.

In the classical analysis for RWs with the scalar Bernoulli coin $p$ mentioned above, the recursions in Eq. (9) yield for $z \rightarrow 1$, i.e., $t \rightarrow \infty$ according to Eq. (2), these three FPs: $\left(A_{\infty}, B_{\infty}, M_{\infty}\right)=\left(0,0, M_{\infty}\right),\left(1-M_{\infty}, 0, M_{\infty}\right)$, or $\left(0,1-M_{\infty}, M_{\infty}\right)$, where $M_{\infty}$ remains as an irrelevant constant that depends on the details. Expanding Eq. (9) to linear order around each FP, the second (third) FP easily yields the ballistic solutions, corresponding to $d_{w}=1$ in Eq. (4), that describes a drift to the left (right) that is expected universally for any $p<1 / 2(p>1 / 2)$. The first FP is more delicate and can be reached only with an unbiased coin, $p=1 / 2$, such that $A \equiv B \rightarrow 0$ and $M \rightarrow M_{\infty}=1$ for $z \rightarrow 1$. At this FP, a naive linearization fails. The self-term $M$ dominates because, in unbiased diffusion, the "range" $L_{k}=2^{k}$ of a $k$-fold renormalized site outgrows the spread of RW at a time $2^{k}$ such that almost all hops remain within that range, making $M_{k} \sim 1$. To subtract this trivial leading behavior, a correlated solution has to be constructed with $A_{k} \equiv B_{k} \sim x_{k} \alpha^{k}$ and $M_{k} \sim 1-y_{k} \alpha^{k}$ for large $k$ and $|\alpha|<1$. Then Eqs. (9) yield $\alpha x_{k+1}=x_{k}^{2} / y_{k}$ and $\alpha y_{k+1}=y_{k}-2 x_{k}^{2} / y_{k}$ with a single FP that self-consistently determines $\frac{x_{\infty}}{y_{\infty}}=\alpha=\frac{1}{2}$. The Jacobian $J_{k}=\frac{\partial\left(x_{k+1}, y_{k+1}\right)}{\partial\left(x_{k}, y_{k}\right)}$, obtained from linearizing these recursions at its FP for $k \rightarrow \infty$, gives $\lambda=4$ as the largest eigenvalue. Thus, for rescaling size $L_{k+1}=2 L_{k}$, time rescales as $t_{k+1}=\lambda t_{k}$, as implied by the Tauberian theorems $[47,49,58]$. Then, from $t_{k} \sim L_{k}^{d_{w}}$, we obtain $d_{w}=\log _{2} \lambda=2$ for the diffusive solution.

The corresponding asymptotic solution of a QW with RG, in which case Eq. (9) is a set of matrix recursions, has been explored at length in Refs. [45,48]. Since it will emerge as a special case of the discussion in Sec. V, we defer its consideration until then.

\section{RENORMALIZATION OF WALKS WITH AN ULTRAMETRIC SET OF BARRIERS}

In the preceding section, we have reviewed how to use RG to solve a homogeneous walk problem using a hierarchical approach. Having ignored its translational invariance in our solution affords us the freedom to explore more general, albeit strictly hierarchical problems on the line. As the behavior of QWs in heterogeneous environments is a largely unexplored subject [35], investigating QWs analytically in a model with a spatially varying coin that respects the hierarchical order seems to be a fruitful task.

To that end, we consider position-dependent coins in such a way that all sites of odd index $x$ share the same coin, and so do all sites that are once-, twice-, thrice-, $\ldots, i$-times divisible by 2. We thus define the binary decomposition $x(i, j)=2^{i}(2 j+$ 1) with a hierarchy index, $i \geqslant 0$, and running index, $-\infty<$ $j<\infty$, providing a unique, one-to-one relation between $x(\neq$ $0)$ and the pair $(i, j)$. Then all sites $x(\neq 0)$ that share the same value of $i$ have an identical coin for all $j$, i.e., $\mathcal{C}_{x(i, j)}=\mathcal{C}_{i}$.

In principle, there are many dynamically interesting sequences of coins that could be defined on such a hierarchy. In the following, we will focus on the case representing progressively more confining barriers, which has been studied classically as a model for glassy behavior. For instance, similar classical models have been proposed for slow relaxation and aging [36-41]. As even homogeneous QWs have been shown to exhibit peculiar localization behavior [13,21], it is an interesting question whether such barriers induce novel quantum effects that are absent classically. 


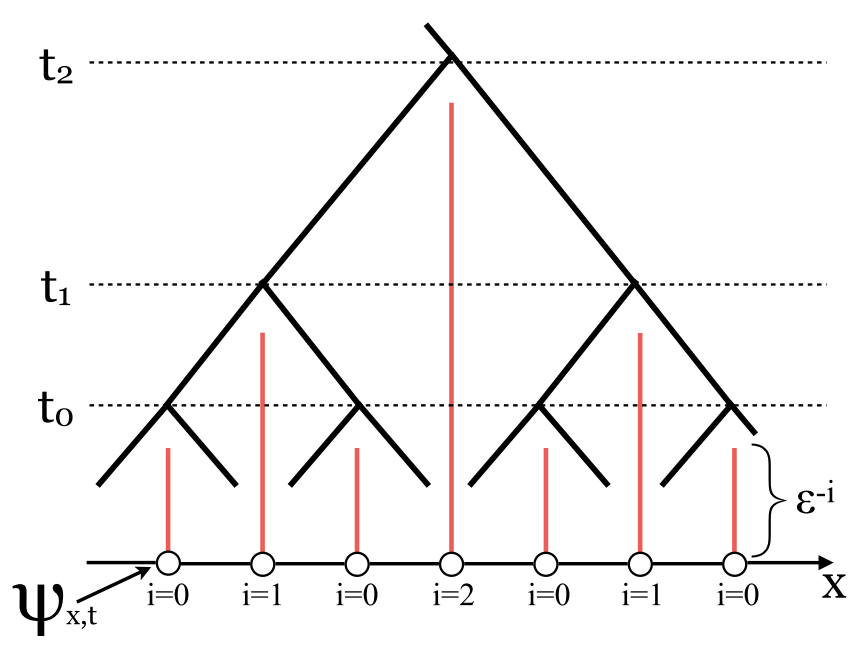

FIG. 1. Depiction of the hierarchical set of barriers (red vertical lines) of relative reflectivity $\epsilon^{-i}$ for $0<\epsilon<1$ and hierarchical index $i$ on a 1D line, as implemented in Eq. (21) for the classical walk and in Eq. (21) for the quantum walk, generating an ultrametrically arranged set of domains (illustrated by a tree) with a hierarchy of characteristic timescales $t_{i}$ for escape.

A hierarchy of barriers arises when the sequence of such coins becomes ever more reflective for a walker trying to transition through the respective site. Then the walker gets confined in a treelike ultrametric set of domains with vastly varying timescales for exit. Two neighboring domains at level $i$ form a larger domain at level $i+1$, and so on, from which an ultrametric hierarchy emerges. Barriers between such domains are depicted in Fig. 1.

It is straightforward to adapt the discussion of the RG for the homogeneous case in Sec. II D to the propagator in Eq. (5) with position-dependent hopping operators. This generalizes the master equation in Eq. (7) to

$$
\bar{\psi}_{x}=z M_{x} \bar{\psi}_{x}+z A_{x-1} \bar{\psi}_{x-1}+z B_{x+1} \bar{\psi}_{x+1} .
$$

Again, we successively eliminate all sites for which $x$ is an odd number $(i=0)$ and set $x \rightarrow x / 2(i \rightarrow i-1)$, thereby removing an entire hierarchy with every iteration, each with an identical coin $\mathcal{C}_{i}$. Starting at $k=0$ with the "raw" hopping operators $A_{i}^{(0)}=z A_{x(i, j)}, B_{i}^{(0)}=z B_{x(i, j)}$, and $M_{i}^{(0)}=z M_{x(i, j)} \equiv$ 0 , step by step for $k=0,1,2, \ldots$, the master equation remains self-similar in form by identifying the renormalized hopping operators $A_{i}^{(k)}, B_{i}^{(k)}, M_{i}^{(k)}$. In analogy to Eq. (8), we focus on a site $x=x\left(i_{+}, j\right)$ with $i_{+} \geqslant 2$, which pertains to every fourth site on the line. Note that all sites \pm 1 or \pm 3 hops removed from $x$ are of odd index $(i=0)$, while those \pm 2 hops removed must have $i=1$. At RG step $k$ we have

$$
\begin{aligned}
\bar{\psi}_{x-1} & =M_{0}^{(k)} \bar{\psi}_{x-1}+A_{i_{+}}^{(k)} \bar{\psi}_{x}+B_{1}^{(k)} \bar{\psi}_{x-2}, \\
\bar{\psi}_{x} & =M_{i_{+}}^{(k)} \bar{\psi}_{x}+A_{0}^{(k)} \bar{\psi}_{x+1}+B_{0}^{(k)} \bar{\psi}_{x-1}, \\
\bar{\psi}_{x+1} & =M_{0}^{(k)} \bar{\psi}_{x+1}+A_{1}^{(k)} \bar{\psi}_{x+2}+B_{i_{+}}^{(k)} \bar{\psi}_{x}, \\
\bar{\psi}_{x+2} & =M_{1}^{(k)} \bar{\psi}_{x+2}+A_{0}^{(k)} \bar{\psi}_{x+3}+B_{0}^{(k)} \bar{\psi}_{x+1} .
\end{aligned}
$$

Solving this linear system for the even sites $x, x \pm 2, x \pm 4$, etc., yields

$$
\begin{aligned}
\bar{\psi}_{x} & =M_{i_{+}-1}^{(k+1)} \bar{\psi}_{x}+A_{0}^{(k+1)} \bar{\psi}_{x+2}+B_{0}^{(k+1)} \bar{\psi}_{x-2}, \\
\bar{\psi}_{x+2} & =M_{0}^{(k+1)} \bar{\psi}_{x+2}+A_{i_{+}-1}^{(k+1)} \bar{\psi}_{x+4}+B_{i_{+}-1}^{(k+1)} \bar{\psi}_{x},
\end{aligned}
$$

and so on. (We have ignored localized ICs on some site, which have no effect on the RG, similar to Sec. II D.) Matching the solutions for those even sites to Eq. (13), we can read off the RG recursions for all $i>0$ :

$$
\begin{aligned}
A_{i-1}^{(k+1)}= & A_{0}^{(k)}\left[\mathbb{I}-M_{0}^{(k)}\right]^{-1} A_{i}^{(k)} \\
B_{i-1}^{(k+1)}= & B_{0}^{(k)}\left[\mathbb{I}-M_{0}^{(k)}\right]^{-1} B_{i}^{(k)} \\
M_{i-1}^{(k+1)}= & M_{i}^{(k)}+A_{0}^{(k)}\left[\mathbb{I}-M_{0}^{(k)}\right]^{-1} B_{i}^{(k)} \\
& +B_{0}^{(k)}\left[\mathbb{I}-M_{0}^{(k)}\right]^{-1} A_{i}^{(k)} .
\end{aligned}
$$

Those RG steps of decimation are illustrated in Fig. 2. Note the close resemblance with Eq. (9), to which Eq. (14) reduces when the (lower) hierarchy indices are removed and the (upper) indices $(k)$ and $(k+1)$ mark unprimed and primed operators, respectively.

Amazingly, we can simplify Eq. (14) even further and entirely eliminate the hierarchy index $i$ : If we define the $k$ th renormalized shift matrices $S_{k}^{\{A, B, M\}}$ via

$$
\{A, B, M\}_{i}^{(k)}=S_{k}^{\{A, B, M\}} \mathcal{C}_{i+k},
$$

which matches the definitions above for the unrenormalized systems at $k=0$. In fact, at $k=0$, all shift matrices are given by Eq. (6), independent of position or hierarchy. Then the $S_{k}^{\{A, B, M\}}$ remain $i$-independent for all $k \geqslant 0$. When inserted into Eqs. (14), they satisfy the recursions,

$$
\begin{aligned}
S_{k+1}^{\{A, B\}}= & S_{k}^{\{A, B\}}\left(\mathcal{C}_{k}^{-1}-S_{k}^{M}\right)^{-1} S_{k}^{\{A, B\}} \\
S_{k+1}^{M}= & S_{k}^{M}+S_{k}^{A}\left(\mathcal{C}_{k}^{-1}-S_{k}^{M}\right)^{-1} S_{k}^{B} \\
& +S_{k}^{B}\left(\mathcal{C}_{k}^{-1}-S_{k}^{M}\right)^{-1} S_{k}^{A},
\end{aligned}
$$

which instead have an explicit $k$ dependence via the inverse coins $\mathcal{C}_{k}^{-1}$ of the $k$ th hierarchy [59].

Up to this point, there was no need to specify whether this ultra-walk is classical or quantum. For RWs, it would seem that we could simply choose scalar Bernoulli coins $p_{x}$ again, such that $A_{x}=p_{x}, B_{x}=1-p_{x}$, and $M_{x}=0$, say, to satisfy the local conservation of probability, $A_{x}+B_{x}+M_{x}=$ 1. However, as the nonlocal construction of Maritan and Stella [38] illustrates, it is not possible to obtain a walk model with ultrametric barriers employing merely such plain scalar coins. Here we present an alternative version of that ultradiffusion model that is intuitive and has greater conceptual simplicity at the expense of adding an internal (coin) degree of freedom. Such a construction [48] is easily recognized as a secondorder Markov process [46] or a persistent random walk (PRW) [47]. It has the added benefit of being completely analogous to the above construction of QWs: We merely replace the unitary coin by a stochastic coin $\mathcal{C}_{x}$, and then the sum $A_{x}+B_{x}+M_{x}$ is also unitary or stochastic, respectively. 

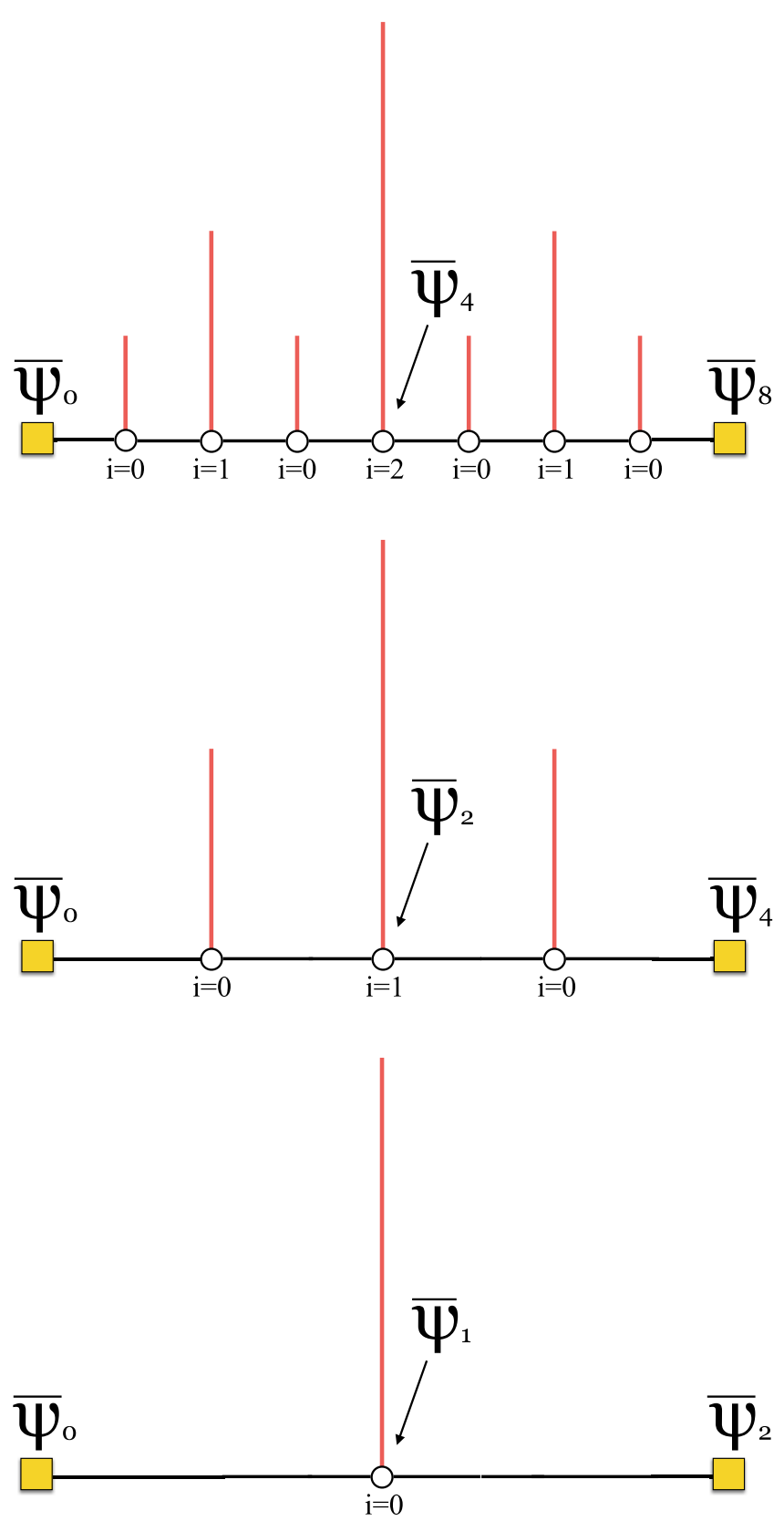

FIG. 2. Last three RG steps $k=l-3, l-2$, and $l-1$, in an ultra-walk with absorbing walls (yellow squares). Note that for each RG step $k \rightarrow k+1$, the remaining hierarchy indices $i$ for sites $x$ go from $i \rightarrow i-1$, as reflected in Eq. (14). Then for all sites $x^{(k)} \rightarrow$ $x^{(k+1)}=\frac{x^{(k)}}{2}$. The final step $k=l-1 \rightarrow l$ is described by Eqs. (16)(17), assuming that the walk started at the central site, $x=x^{(0)}=$ $2^{l-1}=\frac{N-1}{2}$ in a finite system of size $N=2^{l}+1$, originally, i.e., at $x^{(l-1)}=1$.

\section{Absorbing walls}

While the spreading behavior in itself, characterized by the walk dimension $d_{w}$, is the most fundamental property of a walk, other physical properties may be of interest. Another interesting physical quantity is the absorption of a walk at a wall, classically [60-62] or quantum [9]. In particular, since the spreading behavior merely measures the dynamics of that part of the walk which actually moves, determining the absorption of the walk at a confining wall distant from the initial site provides information about how much of the weight of the walk ever reaches the wall. In turn, if that absorption does not become unity, some weight must have become localized within a bounded domain within those walls. For classical diffusion in a simply connected domain that would seem unphysical. However, QWs do exhibit such localization behavior, even in the absence of disorder [13,2023]. Of course, absorption is merely an indirect measure of localization, yet sufficient to ascertain the lack of it.

As a specific situation for such a setting, it is most convenient within the formalism we have developed to consider a walk between two absorbing walls of separation $N=2^{l}+1$, equidistant from the starting site $x_{0}=2^{l-1}$, as illustrated in Fig. 2. Note that in this $1 \mathrm{D}$ geometry, these walls completely confine the walk. As the wall sites $x=0$ and $x=2^{l}$ are fully absorbing, there is no flow out of those sites, and at the end of $l-1 \mathrm{RG}$ steps Eqs. (12) reduce to

$$
\begin{aligned}
& \bar{\psi}_{0}=A_{0}^{(l-1)} \bar{\psi}_{1}, \\
& \bar{\psi}_{1}=M_{0}^{(l-1)} \bar{\psi}_{1}+\psi_{\mathrm{IC}}, \\
& \bar{\psi}_{2}=B_{0}^{(l-1)} \bar{\psi}_{1} .
\end{aligned}
$$

Thus, for either wall it is

$$
\bar{\psi}_{\{0,2\}}=S_{l-1}^{\{A, B\}}\left(\mathcal{C}_{l-1}^{-1}-S_{l-1}^{M}\right)^{-1} \psi_{\mathrm{IC}} .
$$

We will discuss below the RG prediction for the absorption for both RWs and QWs.

\section{SOLUTION OF THE CLASSICAL ULTRA-WALK}

Like for QWs, we will find that the state variable describing PRWs is now a two-component vector $\psi_{x, t}$, which here expresses a memory of the previous step. The physics of classical walks with such memory ("persistence") has been widely studied $[46,47]$. Based on its prior behavior, the upper component $\psi_{x, t}^{+}$refers to a walker with the preference to step to the right in the next time step, and the lower component $\psi_{x, t}^{-}$ indicates a left-hop preference. The value of each component describes the probability of finding a walker at that site $x$ and time $t$ in state " \pm ," and the total probability of finding a walker there, irrespective of preference, is simply the sum of the two, $\rho_{x, t}=\psi_{x, t}^{+}+\psi_{x, t}^{-}$. Ignoring a potential left-right bias here, we consider a walker coming from the left (right) to have a probability $\eta_{x}$ to continue to move right (left), and a probability $1-\eta_{x}$ to reverse direction in the next step. For $\eta_{x}>\frac{1}{2}\left(\eta_{x}<\frac{1}{2}\right)$ the walker exhibits (anti-)persistence and for $\eta_{x}=\frac{1}{2}$ reduces again to an ordinary unbiased RW without memory. The master equations then read

$$
\begin{aligned}
& \bar{\psi}_{x}^{+}=z \eta_{x} \bar{\psi}_{x-1}^{+}+z\left(1-\eta_{x}\right) \bar{\psi}_{x-1}^{-}+\delta_{x, x_{0}} \bar{\psi}_{\mathrm{IC}}^{+}, \\
& \bar{\psi}_{x}^{-}=z\left(1-\eta_{x}\right) \bar{\psi}_{x+1}^{+}+z \eta_{x} \bar{\psi}_{x+1}^{-}+\delta_{x, x_{0}} \bar{\psi}_{\mathrm{IC}}^{-},
\end{aligned}
$$

where $\bar{\psi}_{\text {IC }}\left(\right.$ with $\left.\bar{\psi}_{\text {IC }}^{+}+\bar{\psi}_{\text {IC }}^{-}=1\right)$ represents the ICs of PRWs, which we place again at some site $x_{0}$. Thus, $\bar{\psi}_{x}^{+}\left(\bar{\psi}_{x}^{-}\right)$depends only on hops from its left (right) neighbor; it is that inflow which induces the "+" ("-") state. We can then write Eq. (18) conveniently in matrix notation as a propagator like Eq. (5) 
with [48]

$$
A_{x}=\left(\begin{array}{cc}
\eta_{x} & 1-\eta_{x} \\
0 & 0
\end{array}\right), \quad B_{x}=\left(\begin{array}{cc}
0 & 0 \\
1-\eta_{x} & \eta_{x}
\end{array}\right),
$$

and $M_{x}=0$. As for QWs, we can decompose these matrices further to write them as a combination of a shift and a coin matrix, $\{A, B, M\}_{x}=S^{\{A, B, M\}} \mathcal{C}_{x}$, with the same shift matrices as in Eq. (6). However, here we introduce the stochastic coin matrix

$$
\mathcal{C}_{x}=\left(\begin{array}{cc}
\eta_{x} & 1-\eta_{x} \\
1-\eta_{x} & \eta_{x}
\end{array}\right),
$$

in which each row sums to unity.

\section{A. Ultradiffusion as hierarchically antipersistent walk}

With the same choice of a hierarchically defined coin as in Sec. III, decomposing $x=x(i, j)$, a PRW is renormalized exactly the same way such as to obtain Eq. (15). [Note that $\mathcal{C}_{x}$ in Eq. (20) also has an inverse, except for $\eta_{x}=\frac{1}{2}$, the degenerate case of an ordinary (nonpersistent) walk, which we can safely exclude in the following.] Then it is easy to formulate a simple PRW that is in the same universality class as the ultradiffusion model solved in Ref. [38], by choosing for $0 \leqslant \epsilon \leqslant 1$ and some $\eta_{0}\left(<\frac{1}{2}\right.$, to ensure invertibility of all coins):

$$
\eta_{x(i, j)}=\eta_{i}=\eta_{0} \epsilon^{i} .
$$

For $\epsilon=1$, we expect to recover the ordinary PRW on a homogeneous 1D lattice. Since $\eta_{i}<\frac{1}{2}$ and, indeed, rapidly approaches zero, the walk is increasingly antipersistent, i.e., ever-larger domains form that are bordered by sites $x$ of high hierarchical index $i$, frustrating the walk attempting to leave the domain with an exponentially smaller probability (or higher barriers) with $i$.

Because the RG recursions in Eq. (15) are expressed in terms of matrices, we need to find a parametrization of those recurring shift matrices in terms of scalar variables [48]. After several iterations of Eq. (15), starting from the unrenormalized shift matrices in Eq. (6), a pattern soon appears that can be parametrized as

$$
\begin{aligned}
S_{k}^{A} & =\left(\begin{array}{cc}
a_{k} & 0 \\
0 & 0
\end{array}\right), \quad S_{k}^{B}=\left(\begin{array}{cc}
0 & 0 \\
0 & a_{k}
\end{array}\right), \\
S_{k}^{M} & =\left(\begin{array}{cc}
0 & m_{k} \\
m_{k} & 0
\end{array}\right) .
\end{aligned}
$$

That pattern reproduces itself after a single iteration of Eq. (15) by replacing $k$ with $k+1$ and identifying

$$
\begin{aligned}
a_{k+1} & =\frac{\eta_{k} a_{k}^{2}}{\left(1-m_{k}\right)\left[1-\left(1-2 \eta_{k}\right) m_{k}\right]}, \\
m_{k+1} & =m_{k}+\frac{a_{k}^{2}\left[1-\eta_{k}-\left(1-2 \eta_{k}\right) m_{k}\right]}{\left(1-m_{k}\right)\left[1-\left(1-2 \eta_{k}\right) m_{k}\right]},
\end{aligned}
$$

as the RG flow, with initial conditions $a_{0}=z$ and $m_{0}=0$. Note that this recursion is nonautonomous due to the explicit $k$ dependence via $\eta_{k}$. We could supplement $\eta_{k}$ as a dynamical variable via its recursion $\eta_{k+1}=\epsilon \eta_{k}$ [38] and study fixed points of those three recursions. However, there is a more elegant approach using the transformations

$$
\begin{aligned}
a_{k} & =\frac{\eta_{k}}{1-2 \eta_{k}} \alpha_{k}, \\
m_{k} & =\frac{1-\eta_{k}}{1-2 \eta_{k}}-\frac{\eta_{k}}{1-2 \eta_{k}} \mu_{k},
\end{aligned}
$$

which turn Eqs. (23) into

$$
\begin{aligned}
\alpha_{k+1} & =\frac{1}{\epsilon} \frac{\alpha_{k}^{2}}{\mu_{k}^{2}-1}, \\
\mu_{k+1} & =\left(1-\frac{1}{\epsilon}\right)+\frac{1}{\epsilon} \mu_{k}\left(1-\frac{\alpha_{k}^{2}}{\mu_{k}^{2}-1}\right),
\end{aligned}
$$

where we have assumed $\frac{\eta_{k}\left(1-2 \eta_{k+1}\right)}{\eta_{k+1}\left(1-2 \eta_{k}\right)} \sim \frac{1}{\epsilon}$ and $\frac{1-\eta_{k+1}}{\eta_{k+1}}-$ $\frac{\left(1-\eta_{k}\right)\left(1-2 \eta_{k+1}\right)}{\eta_{k+1}\left(1-2 \eta_{k}\right)} \sim 1-\frac{1}{\epsilon}$, which is justified for $\eta_{k}$ in Eq. (21) at large $k$ for $\epsilon<1$ but trivially holds for the homogeneous walk at $\epsilon=1$ also. Now, the RG flow in Eq. (25) is purely autonomous but depends nontrivially on the parameter $\epsilon$ that characterizes the strength of the ultrametric barriers. This flow has two obvious fixed points, at $\alpha_{\infty}=\frac{1}{\epsilon}-2$ and $\mu_{\infty}=\frac{1}{\epsilon}-$ 1 , and at $\alpha_{\infty}=\frac{1}{\epsilon}-\epsilon$ and $\mu_{\infty}=-\frac{1}{\epsilon}$. The second one cannot be reached by any physical initial condition of the flow, as $\mu_{\infty}<0$. The first fixed point is physical for $0 \leqslant \epsilon \leqslant \frac{1}{2}$, where the largest eigenvalue of the Jacobian of the flow in Eq. (25) is $\lambda=\frac{2}{\epsilon}$, which reproduces the anomalous walk dimension,

$$
d_{w}=1-\log _{2} \epsilon,
$$

found in Ref. [38]. When $\epsilon \rightarrow \frac{1}{2}, d_{w} \rightarrow 2$ and the effect of the barriers becomes irrelevant such that ordinary diffusion ensues for all $\frac{1}{2} \leqslant \epsilon \leqslant 1$, making diffusion rather robust against the introduction of such a set of barriers. [In fact, for $\epsilon=1$ only, we can find a closed form solution for all $k$ of the RG flow in Eq. (25), as in Refs. [45,63].] However, to find the fixed point for the diffusive solutions for $\frac{1}{2} \leqslant \epsilon \leqslant 1$ requires a scaling ansatz with $\alpha_{k}=(2 \epsilon)^{-k} x_{k}$ and $\mu_{k}=1+(2 \epsilon)^{-k} y_{k}$ such that

$$
x_{k+1} \sim \frac{x_{k}^{2}}{y_{k}}, \quad y_{k+1} \sim 2 y_{k}-\frac{x_{k}^{2}}{y_{k}},
$$

now independent of $\epsilon$, with fixed point $x_{\infty}=y_{\infty}$ and a Jacobian eigenvalue of $\lambda=4$, i.e., $d_{w}=2$. These results are summarized in Fig. 3.

Finally, we note that replacing the antipersistent hierarchy of coins with a persistent one, easily achieved by replacing $\eta_{x} \rightarrow 1-\eta_{x}$ in Eq. (20), leads again to a purely diffusive walk for all $\epsilon$. The asymptotic analysis for large $k$ with $\epsilon^{k} \rightarrow 0$ now yields the $\epsilon \rightarrow 1$ limit of Eq. (25) even for $\epsilon<1$ that then results in Eq. (27) with $d_{w}=2$. It is easy to see that the physical situation of a persistent hierarchy differs dramatically from the antipersistent one: For large $k$, all coins now become transmissive (i.e., the identity matrix) instead of reflective, leaving mostly the nondiagonal coins at all odd sites $(i=0)$ to institute a simple persistent, and effectively homogeneous, walk that remains in the same universality class as ordinary diffusion [47]. 


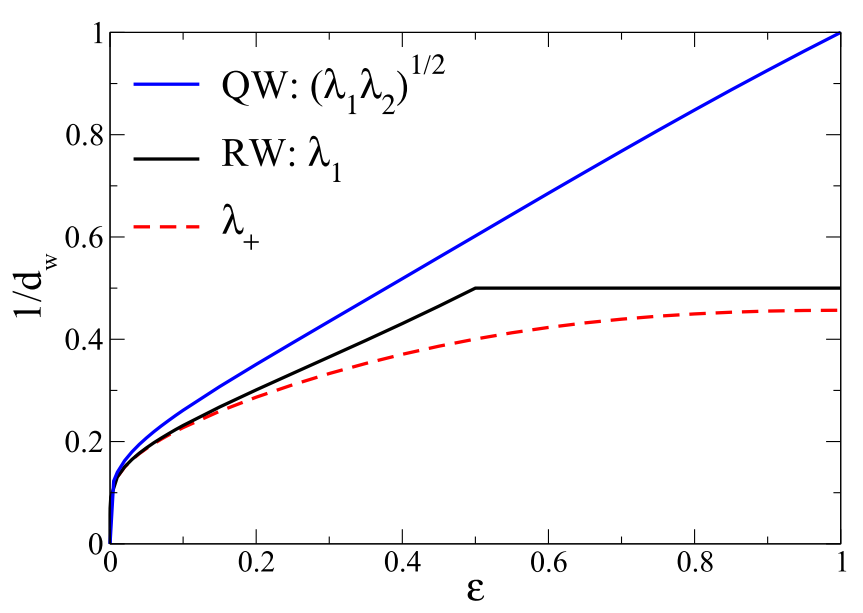

FIG. 3. Plot of the inverse walk dimension $1 / d_{w}$ for the classical (RW) and the quantum walk $(\mathrm{QW})$ with ultrametric barriers as a function of coin parameter $\epsilon$, where smaller $\epsilon$ represents higher barriers between an ultrametrically arrange hierarchy of domains that confine the walk. The black and the blue lines are the respective RG predictions in Eq. (26) and (37). The unphysical prediction from the eigenvalue $\lambda_{+}$in Eq. (35) is shown as red dashed line.

\section{B. Classical walk with absorbing walls}

Evaluation of Eq. (17) for the geometry of Fig. 2 using the coin in Eq. (20) and the RG parametrization in Eq. (22) after $k=l$ RG steps, then taking $l \rightarrow \infty$, we readily obtain

$$
\begin{aligned}
\bar{\psi}_{\{0,2\}} & =\frac{\alpha_{\infty}}{\mu_{\infty}^{2}-1} S^{\{A, B\}}\left(\begin{array}{cc}
1 & \mu_{\infty} \\
\mu_{\infty} & 1
\end{array}\right) \psi_{\mathrm{IC}}, \\
& =S^{\{A, B\}}\left(\begin{array}{cc}
\epsilon & 1-\epsilon \\
1-\epsilon & \epsilon
\end{array}\right) \psi_{\mathrm{IC}},
\end{aligned}
$$

with $S^{\{A, B\}}$ given in Eq. (6). In PRWs the norm of a state vector is simply the sum of its (certainly non-negative) components, $\left\|\psi_{x, t}\right\| \stackrel{\text { def }}{=}\left(\begin{array}{l}1 \\ 1\end{array}\right) \circ \psi_{x, t}$. Then, for an absorbing site $x$, the absorption there is $F_{x}=\sum_{t=0}^{\infty}\left|\psi_{x, t}\right|=\left\|\bar{\psi}_{x}(z=1)\right\|$. With $S^{A}+$ $S^{B}=\mathbb{I}$, we finally get for the total (combined) absorption:

$$
F_{0+2}=\left\|\bar{\psi}_{0}+\bar{\psi}_{2}\right\|=\left\|\psi_{\mathrm{IC}}\right\|=1,
$$

since $\psi_{\mathrm{IC}}$ is normed to unity, of course. Thus, for any size barrier $\epsilon$ and any system size, any walk started in the middle will eventually get absorbed with certainty! For a classical walk on the $1 \mathrm{D}$ line, we would have expected this, due to Polya's theorem [44], even for a heterogeneous environment.

\section{SOLUTION OF THE QUANTUM ULTRA-WALK}

To design a quantum analog to the classical PRW on an ultrametric set of barriers, specified by Eq. (20), we consider the real, unitary quantum coins

$$
\mathcal{C}_{i}=\left(\begin{array}{cc}
\sin \eta_{i} & \cos \eta_{i} \\
\cos \eta_{i} & -\sin \eta_{i}
\end{array}\right), \quad \eta_{i}=\eta_{0} \epsilon^{i} \quad(0<\epsilon \leqslant 1),
$$

although many other interesting choices may exist [22]. Note that for $\epsilon=1$, this reproduces a homogeneous 1D QW [48]. However, for $\epsilon<1$, the coins become increasingly resistant to transit, with $\eta_{i} \rightarrow 0$ for $i \rightarrow \infty$, blocking the transition through sites $x$ of higher index $i$, no matter from which direction those sites are approached.

As for the classical case, evolving the recursions in Eq. (15) with this coin for a few iterations from the unrenormalized values, already after one iteration a recurring pattern emerges that suggest the ansatz

$$
\begin{aligned}
S_{k}^{A} & =\left(\begin{array}{cc}
a_{k} & 0 \\
0 & 0
\end{array}\right), \quad S_{k}^{B}=\left(\begin{array}{cc}
0 & 0 \\
0 & -a_{k}
\end{array}\right), \\
S_{k}^{M} & =\left(\begin{array}{cc}
0 & m_{k} \\
m_{k} & 0
\end{array}\right),
\end{aligned}
$$

amazingly similar to the classical case in Eq. (22). When iterated, the RG recursions in Eq. (15) for the scalar parametrization with $a_{k}$ and $m_{k}$ closes after each iteration for

$$
\begin{aligned}
a_{k+1} & =\frac{a_{k}^{2} \sin \eta_{k}}{1-2 m_{k} \cos \eta_{k}+m_{k}^{2}}, \\
m_{k+1} & =m_{k}+\frac{\left(m_{k}-\cos \eta_{k}\right) a_{k}^{2}}{1-2 m_{k} \cos \eta_{k}+m_{k}^{2}},
\end{aligned}
$$

with $a_{k=1}=z^{2} \sin \eta_{0}, m_{k=1}=z^{2} \cos \eta_{0}$, and $\eta_{1}=\eta_{0} \epsilon$ as initial conditions. [Only the first step, from $k=0$ to $k=1$, does not fit this pattern.] Note the striking similarity of these recursions to those for PRWs in PRWs in Eq. (23). Like those, Eq. (32) is nonautonomous. Analogous to Eq. (24), we can find an elegant transformation,

$$
a_{k}=\alpha_{k} \sin \eta_{k}, \quad m_{k}=\cos \eta_{k}-\mu_{k} \sin \eta_{k},
$$

that turns Eq. (32) into

$$
\begin{aligned}
\alpha_{k+1} & \sim \frac{1}{\epsilon} \frac{\alpha_{k}^{2}}{\mu_{k}^{2}+1}, \\
\mu_{k+1} & \sim \frac{1}{\epsilon} \mu_{k}\left(1+\frac{\alpha_{k}^{2}}{\mu_{k}^{2}+1}\right)+\frac{1}{2} \eta_{k}\left(\epsilon-\frac{1}{\epsilon}\right),
\end{aligned}
$$

where we have approximated $\frac{\sin \eta_{k+1}}{\sin \eta_{k}} \sim \epsilon$ and $\left(\cos \eta_{k}-\cos \eta_{k+1}\right) / \sin \eta_{k+1} \sim \frac{1}{2} \eta_{k}(\epsilon-1 / \epsilon), \quad$ to within exponentially small corrections in $k$ for $\epsilon<1$, and trivially correct for $\epsilon=1$.

If we were to consider neglecting the last term in Eq. (34), that is exponentially small for $\epsilon<1$, we find a fixed point at $\alpha_{\infty}=1-\frac{1}{\epsilon}$ and $\mu_{\infty}= \pm i \sqrt{1-\frac{1}{\epsilon}+\frac{1}{\epsilon^{2}}}$. Having $\alpha_{\infty}<0$ imposes no restriction, since the definition of $a_{k}$ in Eq. (31) is invariant under $a_{k} \rightarrow-a_{k}$. Having imaginary $\mu_{\infty}$ could be expected as a small correction off the real axis to $m_{\infty}=1$ in a quantum problem. The associated eigenvalues are very interesting, with

$$
\lambda_{ \pm}=\left(\frac{1}{\epsilon}+\frac{1}{2}+\epsilon\right) \pm \sqrt{\left(\frac{1}{\epsilon}+\frac{1}{2}+\epsilon\right)^{2}-2}
$$

of which only $\lambda_{+}>1$ for $0<\epsilon<1$. In fact, it reproduces the eigenvalues found for a corresponding tight-binding spectrum considered in Ref. [40]. As shown in Fig. 3, it meets up with the classical result for $d_{w}$ really well for $\epsilon \rightarrow 0$. However, it generally predicts a slower spread than even the classical walk throughout. Closer inspection of the boundary layer [64] at $\epsilon \rightarrow 1$ shows that it is actually a very subtle extension of 
the (subdominant) diffusive solution found for the ordinary 1D QW discussed in Ref. [48]. While $\lambda_{+} \rightarrow(5+\sqrt{17}) / 2 \approx$ $4.56 \ldots$ for $\epsilon \rightarrow 1$, this fixed point is actually not valid for $\epsilon=1$, since $\mu_{\infty} \rightarrow \pm i$ and $\alpha_{\infty} \rightarrow 0$, for which the recursions in Eq. (34) are singular. Resolving that singularity with a scaling ansatz reproduces the diffusive solution with $\lambda=4$ to which $\lambda_{+}$discontinuously connects.

To reveal the physically relevant scaling of QW, we argue as follows: Without the last term in Eq. (34), there is also a fixed point values of $\alpha_{\infty}=\epsilon$ and $\mu_{\infty}=0$, which in turn is inconsistent with dropping even an exponentially small term, however. Rather, we retain the term and apply the ansatz $\mu_{k}=$ $\eta_{k} v_{k} \ll 1$ with $v_{k}$ finite to turn all of Eq. (34) autonomous:

$$
\begin{aligned}
\alpha_{k+1} & \sim \frac{\alpha_{k}^{2}}{\epsilon}, \\
v_{k+1} & \sim \frac{v_{k}}{\epsilon^{2}}\left(1+\alpha_{k}^{2}\right)-\frac{1-\epsilon^{2}}{2 \epsilon^{2}} .
\end{aligned}
$$

The flow in Eq. (36) has a fixed point at $\alpha_{\infty}=\epsilon$ and $v_{\infty}=$ $\frac{1}{2}\left(1-\epsilon^{2}\right)$. Its Jacobian eigenvalues are $\lambda_{1}=1+\epsilon^{-2}$ and $\lambda_{2}=2$, i.e., $\lambda_{1} \geqslant \lambda_{2}>1$ for $0 \leqslant \epsilon \leqslant 1$. For a classical walk with a stochastic master equation, the leading eigenvalue $\lambda_{1}$ suffices to describe the walk dimension $d_{w}=\log _{2} \lambda_{1}$ [49]. However, it has been shown [45,50] that the unitarity constraint imposed on the master equation for $\mathrm{QW}$ also requires physical observables to be unitary. Yet the renormalized parameters $a_{k}$ and $m_{k}$ are not, and their poles move with $k$ both, tangentially and radially, to the unit circle in the complex $z$ plane, while the poles of actual observables only move tangentially on the circle; see Eq. (3). Detailed analysis shows [50] that the largest,dominant eigenvalue, $\lambda_{1}$, describes only the tangential movement of poles and, thus, must be ignored [65]. Instead, the tangential movement of poles is found to be described by the geometric mean of first and second eigenvalue, $\sqrt{\lambda_{1} \lambda_{2}}$. Accordingly, we conclude that

$$
d_{w}^{Q}=\log _{2} \sqrt{\lambda_{1} \lambda_{2}}=\frac{1}{2}+\frac{1}{2} \log _{2}\left(1+\epsilon^{-2}\right) .
$$

This walk dimension, also shown in Fig. 3, has all the characteristics of being physical, as it is ballistic $\left(d_{w}=1\right)$ for the homogeneous 1D QW at $\epsilon \rightarrow 1$, and it diverges for diverging barrier heights, $\epsilon \rightarrow 0$, where to leading order it matches up with the classical result, $d_{w} \sim-\log _{2} \epsilon$. It also predicts the fastest spread for QWs compared to RWs or the fixed point leading to Eq. (35). Note also that with even a minute disturbance of homogeneity, i.e., barriers for arbitrarily small $1-\epsilon$, the QW ceases to be ballistic.

While it is easy to simulate QWs for any $\epsilon$, it remains a challenge to interpret the data, in particular, to test Eq. (37). Unlike for RWs, the deterministic, unitary evolution of QWs does not provide for much of a stochastic variation over which repeated walks could be averaged. Additionally, sometimes dramatic changes in the behavior of a walk occur, especially near large barriers. Both of these effects are on display in Fig. 4, showing the PDF of QWs with $\epsilon=\frac{1}{2}$, rescaled according to Eq. (4). Large spatial fluctuations, and ever more pronounced jumps near larger barriers, make it difficult to collapse the data with any accuracy. However, asymptotically such a collapse with $d_{w}=1.661$, as Eq. (37) provides, seems plausible.

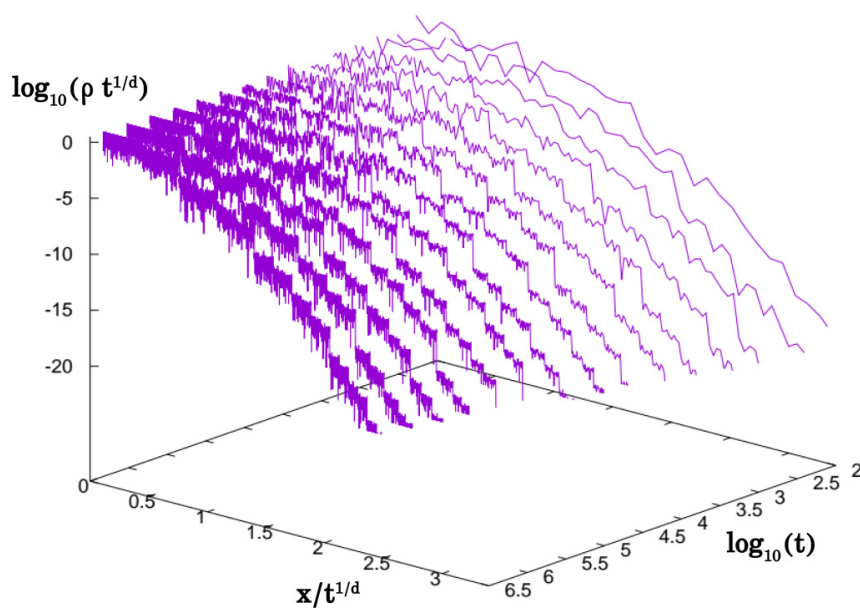

FIG. 4. Plot of the PDF $\rho(x, t)$ as a function of pseudovelocity $x / t^{1 / d_{w}}$ [see Eq. (4)] for a range of times $t$ after the initiation of the walk at $x=0$. (Only $x \geqslant 0$ is shown.) In these simulations, we set $\epsilon=\frac{1}{2}$ so that $d_{w}=1.661$, according to Eq. (37).

\section{Quantum walk with absorbing walls}

Unlike for the classical case discussed in Sec. IV B, where the total absorption amounts conveniently to taking a local limit for $z \rightarrow 1$ on the Laplace transform of the site amplitude, evaluating the adsorption in QWs is surprisingly involved, in comparison. Due to unitarity, the adsorption sums up the square modulus of site amplitudes, which correspond to contour integrals over the entire unit circle in the complex $z$ plane for the modulus of their Laplace transforms,

$$
F_{0}=\sum_{t=0}^{\infty}\left|\psi_{0, t}\right|^{2}=\oint \frac{d z}{2 \pi i z}\left|\bar{\psi}_{0}(z)\right|^{2} .
$$

Such an integral is readily, albeit strenuously, evaluated for the simple case of a homogeneous QW $(\epsilon=1)$, using the nonlinear Riemann-Lebesgue lemma [9]. However, for $\epsilon<$ 1 we have only the local asymptotic evaluation of the RG recursions in Eq. (32) available. While, for instance, $\bar{\psi}_{0}(z)$ in Eq. (38) is a functional of the hopping operators, inserting their asymptotic form for a local expansion of the integral does not appear to be sufficient to obtain the absorption.

As mentioned above, resorting instead to a direct simulation of QWs shows that it is difficult to extract the scaling for moments of the walk to, say, verify the walk dimension in Eq. (37) with any reasonable accuracy. The irregular pattern of reflecting barriers leads to very noisy probability densities. However, it is quite easy to convince oneself, starting from very small systems and progressively doubling their size, that the total absorption remains exactly unity throughout for even the smallest values of $\epsilon$. Thus, there appears to be no localization in the interior of the system; all of the weight of a QW eventually reaches a wall!

\section{CONCLUSIONS}

The ultra-walk provides an exactly solvable model of a walk, either classical or quantum, with tunable spatial heterogeneity. For the classical case, we reproduce previous results by alternative means, using a second-order Markov 
process that closely resembles the coined QW in form. For the discrete-time QW, we obtain results over the entire range of heterogeneity, with walk dimensions ranging from $d_{w}^{\mathrm{QW}}=1$ to infinity. Thus, while the RW is quite robust against the introduction of those barriers, even the smallest amount of inhomogeneity changes the asymptotic behavior of QWs. However, numerical verification of these results for QWs is difficult to obtain, due to the strong hierarchical nature. Focusing on the absorption problem at walls that recede from the starting site with increasing system size, we can at least ascertain, as in the classical case, that there is no localization, even for the most extreme heterogeneity. Future work will focus on the asymptotic evaluation of observables, like the absorption in Eq. (38), that are defined via a complex integration for QWs. Absorption, transit, and first passage problems of that sort are fundamental to any transport problem, classical or quantum $[49,66]$.

\section{ACKNOWLEDGMENTS}

S.B. acknowledges financial support during the preparation of this manuscript from the U.S. National Science Foundation through grant DMR-1207431 and from CNPq through the "Ciência sem Fronteiras" program, and he thanks Renato Portugal, Stefan Falkner, and Shanshan Li for many helpful discussions and LNCC for its hospitality.
[1] R. Portugal, Quantum Walks and Search Algorithms (Springer, Berlin, 2013).

[2] L. K. Grover, Phys. Rev. Lett. 79, 325 (1997).

[3] N. Shenvi, J. Kempe, and K. B. Whaley, Phys. Rev. A 67, 052307 (2003).

[4] A. Ambainis, J. Kempe, and A. Rivosh, in Proceedings of the Sixteenth Annual ACM-SIAM Symposium on Discrete Algorithms, SODA '05 (Society for Industrial and Applied Mathematics, Philadelphia, 2005), pp. 1099-1108.

[5] S. Boettcher, S. Li, T. D. Fernandes, and R. Portugal, Phys. Rev. A 98, 012320 (2018).

[6] A. M. Childs and J. Goldstone, Phys. Rev. A 70, 022314 (2004).

[7] J. Kempe, Contemp. Phys. 44, 307 (2003).

[8] E. Venegas-Andraca, Quantum Inf. Process. 11, 1015 (2012).

[9] A. Ambainis, E. Bach, A. Nayak, A. Vishwanath, and J. Watrous, Proceedings of the Thirty-Third Annual ACM Symposium on Theory of Computing (ACM, New York, 2001), pp. $37-49$.

[10] N. Konno, Quantum Inf. Process 1, 345 (2002).

[11] N. Inui, Y. Konishi, and N. Konno, Phys. Rev. A 69, 052323 (2004)

[12] E. Bach, S. Coppersmith, M. P. Goldschen, R. Joynt, and J. Watrous, J. Comput. Syst. Sci. 69, 562 (2004).

[13] N. Inui, N. Konno, and E. Segawa, Phys. Rev. E 72, 056112 (2005).

[14] T. D. Mackay, S. D. Bartlett, L. T. Stephenson, and B. C. Sanders, J. Phys. A: Math. Gen. 35, 2745 (2002).

[15] F. L. Marquezino, R. Portugal, and S. Boettcher, Phys. Rev. A 87, 012329 (2013).

[16] A. Patel and K. S. Raghunathan, Phys. Rev. A 86, 012332 (2012).

[17] S. Boettcher, S. Falkner, and R. Portugal, Phys. Rev. A 91, 052330 (2015).

[18] F. L. Marquezino, R. Portugal, G. Abal, and R. Donangelo, Phys. Rev. A 77, 042312 (2008).

[19] N. Konno, J. Math. Soc. Jpn. 57, 1179 (2005).

[20] A. Schreiber, K. N. Cassemiro, V. Potoček, A. Gábris, I. Jex, and C. Silberhorn, Phys. Rev. Lett. 106, 180403 (2011).

[21] S. Falkner and S. Boettcher, Phys. Rev. A 90, 012307 (2014).

[22] I. Vakulchyk, M. V. Fistul, P. Qin, and S. Flach, Phys. Rev. B 96, 144204 (2017).
[23] J. Mareš, J. Novotný, and I. Jex, Phys. Rev. A 99, 042129 (2019).

[24] H. B. Perets, Y. Lahini, F. Pozzi, M. Sorel, R. Morandotti, and Y. Silberberg, Phys. Rev. Lett. 100, 170506 (2008).

[25] A. Peruzzo, M. Lobino, J. C. F. Matthews, N. Matsuda, A. Politi, K. Poulios, X.-Q. Zhou, Y. Lahini, N. Ismail, K. Wörhoff et al., Science 329, 1500 (2010).

[26] A. Schreiber, A. Gábris, P. P. Rohde, K. Laiho, M. Štefaňák, V. Potoček, C. Hamilton, I. Jex, and C. Silberhorn, Science 336, 55 (2012)

[27] L. Sansoni, F. Sciarrino, G. Vallone, P. Mataloni, A. Crespi, R. Ramponi, and R. Osellame, Phys. Rev. Lett. 108, 010502 (2012).

[28] A. Crespi, R. Osellame, R. Ramponi, V. Giovannetti, R. Fazio, L. Sansoni, F. D. Nicola, F. Sciarrino, and P. Mataloni, Nat. Photonics 7, 322 (2013).

[29] X. Qiang, T. Loke, A. Montanaro, K. Aungskunsiri, X. Zhou, J. L. O’Brien, J. B. Wang, and J. C. F. Matthews, Nat. Commun. 7, 11511 (2016).

[30] V. V. Ramasesh, E. Flurin, M. Rudner, I. Siddiqi, and N. Y. Yao, Phys. Rev. Lett. 118, 130501 (2017).

[31] C. Figgatt, D. Maslov, K. A. Landsman, N. M. Linke, S. Debnath, and C. Monroe, Nat. Commun. 8, 1918 (2017).

[32] I. Foulger, S. Gnutzmann, and G. Tanner, Phys. Rev. Lett. 112 070504 (2014).

[33] H. Tang, C. D. Franco, Z.-Y. Shi, T.-S. He, Z. Feng, J. Gao, K. Sun, Z.-M. Li, Z.-Q. Jiao, T.-Y. Wang et al., Nat. Photonics 12, 754 (2018).

[34] H. Tang, X.-F. Lin, Z. Feng, J.-Y. Chen, J. Gao, K. Sun, C.-Y. Wang, P.-C. Lai, X.-Y. Xu, Y. Wang et al., Sci. Adv. 4, eaat3174 (2018).

[35] Y. Shikano and H. Katsura, Phys. Rev. E 82, 031122 (2010).

[36] A. T. Ogielski and D. L. Stein, Phys. Rev. Lett. 55, 1634 (1985).

[37] B. Huberman and M. Kerszberg, J. Phys. A 18, L331 (1985).

[38] A. Maritan and A. Stella, J. Phys. A: Math. Gen. 19, L269 (1986).

[39] P. Sibani, Phys. Rev. B 34, 3555 (1986).

[40] H. A. Ceccatto, W. P. Keirstead, and B. A. Huberman, Phys. Rev. A 36, 5509 (1987).

[41] K. H. Hoffmann and P. Sibani, Phys. Rev. A 38, 4261 (1988).

[42] R. K. Pathria, Statistical Mechanics, 2nd ed. (ButterworthHeinemann, Boston, 1996). 
[43] C. Itzykson and J.-M. Drouffe, Statistical Field Theory (Cambridge University Press, Cambridge, 1989).

[44] G. Pólya, Math. Ann. 84, 149 (1921).

[45] S. Boettcher and S. Li, Phys. Rev. A 97, 012309 (2018).

[46] A. Chen and E. Renshaw, J. Appl. Probab. 31, 869 (1994).

[47] G. H. Weiss, Aspects and Applications of the Random Walk (North-Holland, Amsterdam, 1994).

[48] S. Boettcher, S. Falkner, and R. Portugal, J. Phys. Conf. Ser. 473, 012018 (2013).

[49] S. Redner, A Guide to First-Passage Processes (Cambridge University Press, Cambridge, 2001).

[50] S. Boettcher, S. Li, and R. Portugal, J. Phys. A 50, 125302 (2017).

[51] S. Havlin and D. Ben-Avraham, Adv. Phys. 36, 695 (1987).

[52] W. Feller, An Introduction to Probability Theory and its Applications, Vol. 1 (John Wiley, New York, 1966).

[53] J.-P. Bouchaud and A. Georges, Phys. Rep. 195, 127 (1990).

[54] G. Grimmett, S. Janson, and P. F. Scudo, Phys. Rev. E 69, 026119 (2004).

[55] E. Segawa and N. Konno, Int. J. Quant. Inform. 6, 1231 (2008).
[56] N. Konno, in Quantum Potential Theory, Lecture Notes in Mathematics Vol. 1954, edited by U. Franz and M. Schürmann (Springer-Verlag, Heidelberg, 2008), pp. 309-452.

[57] S. Boettcher, S. Falkner, and R. Portugal, Phys. Rev. A 90, 032324 (2014).

[58] B. D. Hughes, Random Walks and Random Environments (Oxford University Press, Oxford, 1996).

[59] We assume that both the unitary and also the stochastic coin have an inverse.

[60] S. Boettcher, Phys. Rev. E 51, 3862 (1995).

[61] S. Boettcher and M. Moshe, Phys. Rev. Lett. 74, 2410 (1995).

[62] C. M. Bender, S. Boettcher, and P. N. Meisinger, Phys. Rev. Lett. 75, 3210 (1995).

[63] S. Boettcher and S. Li, J. Phys. A 48, 415001 (2015).

[64] C. M. Bender and S. A. Orszag, Advanced Mathematical Methods for Scientists and Engineers (McGraw-Hill, New York, 1978).

[65] In the Appendix of Ref. [50] an example was provided that shows by explicit construction how the poles of the nonunitary hopping parameters cancel as soon as these parameters are combined to calculate an observable.

[66] N. G. V. Kampen, Stochastic Processes in Physics and Chemistry (North-Holland, Amsterdam, 1992). 